\title{
Infrared thermography of electroconductive woven textiles
}

\author{
by J.Banaszczyk ${ }^{\mathrm{a}}$, A. Anca ${ }^{\mathrm{b}}, \mathrm{G}$. De Mey ${ }^{\mathrm{a}}$
}

${ }^{a}$ Department of Electronics and Information Systems, Ghent University, Sint Pietersniewstraat 41, 9000 Gent, Belgium

${ }^{b}$ Department of Electrotechnics, Technical University of Cluj-Napoca, C. Daicoviciu str. 15, 400020 Cluj-Napoca, Romania

Abstract

Modelling of the current density in electroconductive textiles is a complicated problem as they cannot be considered homogenous structures with an isotropic current distribution. This anisotropy is mainly the result of the electrical contact resistance between interlacing yarns comprising the textile. It is difficult to measure the contact resistance directly as it is often nonlinear and depends on many factors, however it can by inferred by comparing computer simulations of the power distribution in the textiles with their corresponding IR images.

\section{Introduction}

Electroconductive textiles are a relatively new but promising idea. The range of possible applications they could have in the future is very wide and spreads from medicine [1] to telecommunications [2] and wearable computing [3]. Fibres can be used as conductors, which can be easily incorporated into clothing and form connections between electronic components [4], but they can also be made into textile sheets by means of e.g. weaving or knitting, to form e.g. antennas [2] or electrodes [1]. In order to model the operation of such devices it is necessary to know their internal current distribution.

At first sight any piece of textile can be considered as a thin conducting sheet, which may be described by just one parameter; the sheet conductivity. However, in our previous paper [5] it was shown that this statement no longer holds for electroconductive textiles. Due to the electrical contact resistance between interlacing fibres a sheet of textile cannot always be considered a thin uniform and isotropic conductor.

An electroconductive textile sheet, be it woven or knitted, can be treated as a combination of series and parallel connections of resistors that correspond to the resistivity of the fibres themselves and to the resistance between fibres that are interlaced (contact resistance). The concept is depicted in Figs 1-2. These parameters will depend on numerous external factors such as the temperature, the humidity or the pressure and extension [6][7][8][9]. Furthermore, these may vary as a result of the device heating up during operation. This makes direct measurements of the contact resistance very problematic and that is why it is difficult to create a consistent current distribution model of the textile. Nonetheless, it is possible to infer the contact resistance by comparing the results of our simulations with the temperature distribution patterns we obtained through infrared imaging of actual electroconducting textile samples. A value of contact resistance can be found such that the simulated power distribution patterns closely resemble those of the temperature distribution in the infrared images.
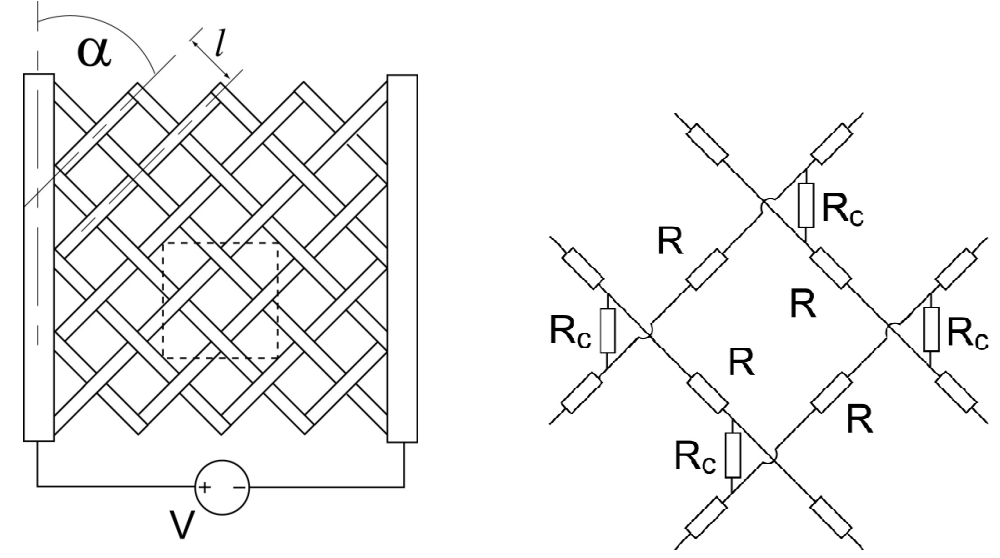

Fig. 1. : Electroconducting textile sheet (left) and its equivalent electrical model (right) 


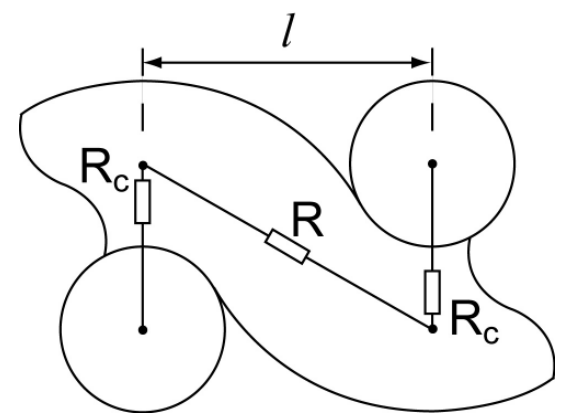

Fig. 2. : Schematics of contact resistance in electroconductive textiles

\section{Simulation program}

The idea of the electrical model is simple in itself, but the main problem is the large size of the circuit, which could reach one hundred thousand elements, making it difficult to simulate using standard electrical circuit simulators. An analytical solution based on Kirchhoff's current and voltage laws is not possible due to the large number of unknowns. Such a solution would become unstable, and the amount of memory required to store the coefficient matrix would be enormous. Basing on the idea introduced in the previous section it was possible to write a program, which iteratively calculates the current distribution and power density in the modelled electroconductive textile. In each iteration the new potential of every node in the circuit is calculated according to the formula [10]:

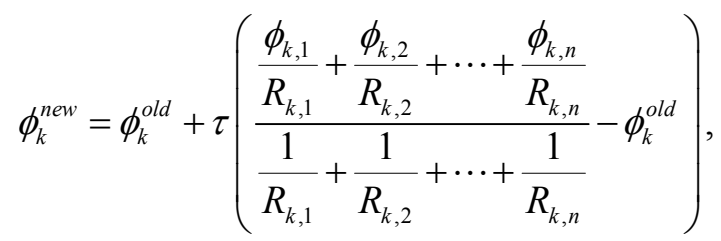

where $\phi_{k}^{\text {new }}$ is the new potential of the $k$-th node after the iteration, $\phi_{k}^{\text {old }}$ is the potential of the $k$-th node before the iteration, $T$ is the relaxation coefficient, $\phi_{k, 1}, \phi_{k, 2} \ldots \phi_{k, n}$ are the potentials of the neighboring nodes of the $k$-th node, and $R_{1}, R_{2} \ldots R_{3}$ are the resistances of the branches connected to the $k$-th node, as shown in Fig. 3 , which can be equal to either $R$ or $R_{c}$. Iterations are repeated until the difference between $\phi_{k}^{\text {new }}$ and $\phi_{k}^{\text {old }}$ for every node in the circuit is smaller than a certain value $\varepsilon$, which can be chosen as a trade-off between the accuracy and the minimum number of iterations required.

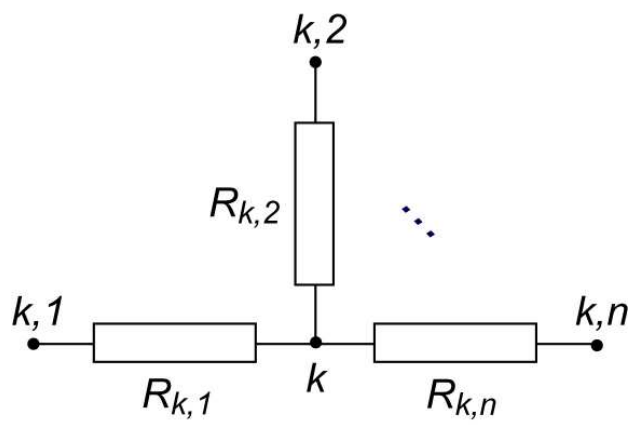

Fig. 3. : Explanation of the iterative method

\section{Infrared imaging vs. simulations}

In our experiment we examined the electrical conductivity of a $140 \times 110 \mathrm{~mm}^{2}$ woven stainless steel sheet (Bekintex), which is shown in Fig. 4. Bekintex is woven out of stainless steel yarns, which have the conductivity of approximately $0.05 \Omega / \mathrm{mm}$. The distance $/$ between the yarns (as depicted in Fig. 2) is about $1 \mathrm{~mm}$. We were interested in finding the value of the contact resistance (denoted $R_{c}$ in Fig. 2) by comparing the heat distribution pattern obtained through infrared thermography to the power distribution patterns generated with our simulation 
program. The current distribution within the textile sheet does not depend directly on the contact resistance $R_{c}$, but rather on the ratio $R_{d} / R$, where $R$ is the partial resistance of a section of length I (see Figs.1-2) of the yarn (in case of Bekintex it is $0.05 \Omega / \mathrm{mm}$ ).

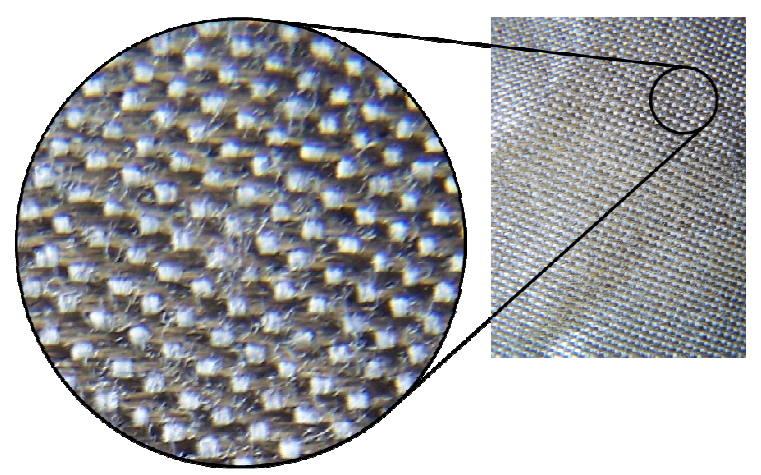

Fig. 4. : Bekintex woven stainless steel textile

The setup presented in Fig. 5 was assembled. A current I $=1.05 \mathrm{~A}$ was fed to the conductive textile using alligator clips and heat distribution patterns were observed using an AGEMA THERMOVISION 900 infrared camera, which are presented in Fig. 6. It is clearly visible, that the current flows along the yarns, which are vertical and horizontal, rather than spreading uniformly in the upper right quarter of the textile. This is a first indication that there exists a significant contact resistance between interlacing yarns. In the image we observe four hot spots. Two of them (on centres of the sides of the fabric) are partially attributed to the contact resistance between the metal clamps feeding the current and the textile and partially to the contact resistance between interlacing yarns. The other two (in the centre and in the upper right corner of the fabric) are related only to the contact resistance between interlacing yarns.

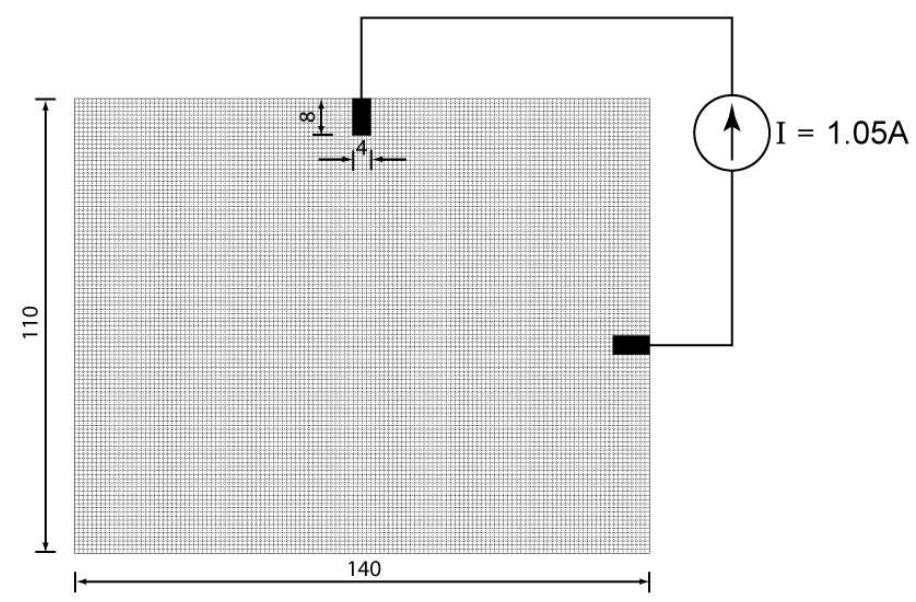

Fig. 5. : Measurement setup (all dimensions in millimeters)

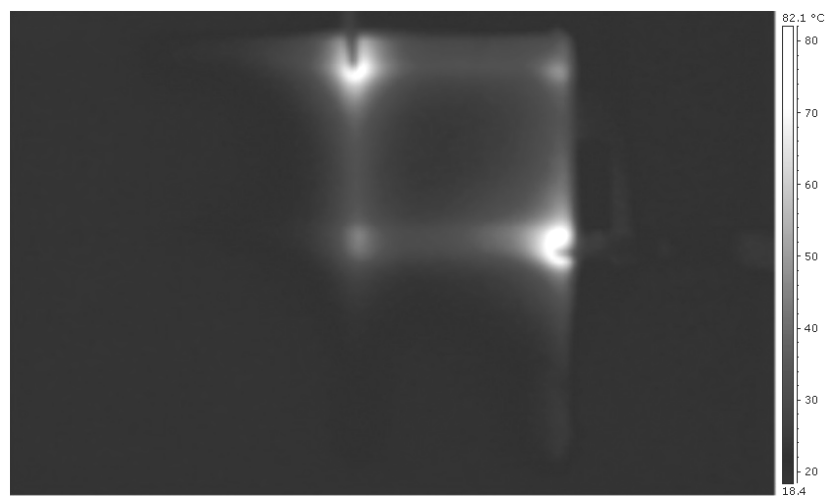

Fig. 6. : Temperature distribution, infrared image 
Using our simulation program we tried to recreate that image by appropriately selecting the value of the contact resistance between interlacing yarns. The first estimation we made was $R_{c}=12 \Omega\left(R_{d} / R=240\right)$. The temperature pattern obtained from the simulation is shown in Fig. 7. Although the two hot spots attributed to the current feeds are visible no hot spots appear in the centre and in the corner of the fabric. This means that the value of $R_{c}$ that we chose was too small and the current spread quite homogenously, without causing a temperature rise within the sheet.

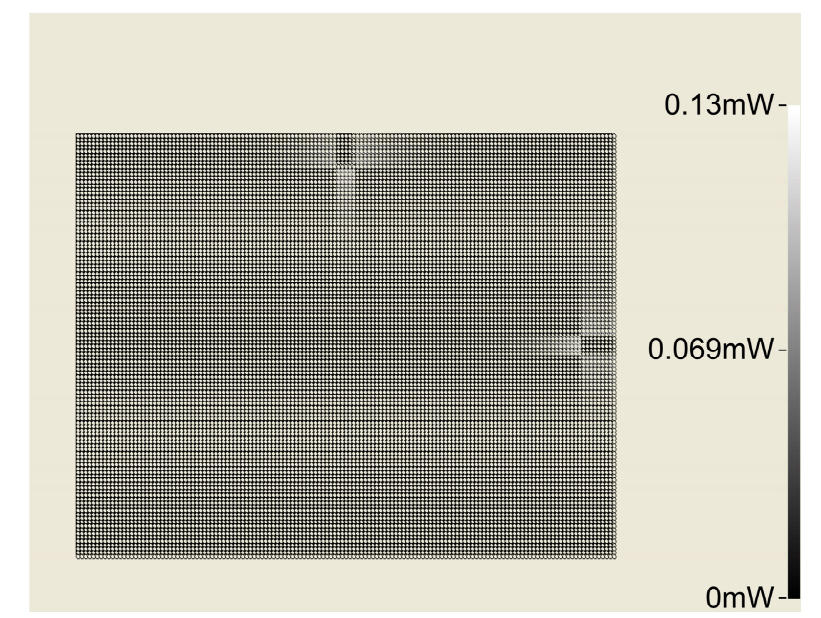

Fig. 7. : Simulated power density, sheet size $=140 \times 110 \mathrm{~mm}^{2}, R_{C} / R=240(12 \Omega)$

We then raised the contact resistance to $100 \Omega\left(R_{d} / R=2000\right)$. The obtained pattern (Fig. 8) is now much more similar to the one in the IR photograph. Four hot spots, as well as the paths taken by the current along the yarns are clearly visible. Thus it can be concluded that the contact resistance in the tested electroconductive textile is high and it influences the current distribution considerably. Such structures cannot be regarded as homogenous.

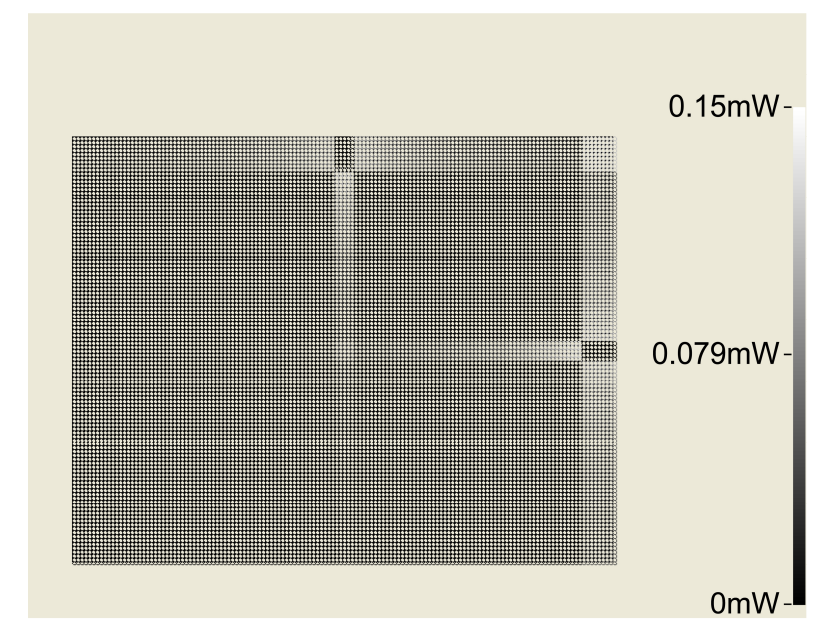

Fig. 8. : Simulated power density, sheet size $=140 \times 110 \mathrm{~mm}^{2}, R_{C} / R=2000(100 \Omega)$

\section{Direct measurements}

In the previous section we estimated the contact resistance to be about $100 \Omega$ basing on the comparison between the infrared measurements and our simulations. In order to validate this result we measured the contact resistance directly using the setup depicted in Fig. 9. Two yarns are intersecting, one of which is loaded with two loads of $2 \mathrm{~g}$ each and thus the total force exerted on the contact point is $4 \mathrm{cN}$. The load was chosen in such a way that a constant contact between the yarns is provided, resembling that in actual textiles. Current is fed to the yarns with alligator clamps and the voltage drop on the contact point is measured as shown in Fig. 9. If we assume that the internal impedance $R_{V}$ of the voltmeter is sufficiently large the current $I_{2}$ is negligible and then $I_{1}=$ 
$I_{c}$, thus the voltage drop measured by the voltmeter will be in fact the voltage drop on the contact resistance $R_{c}$ itself.
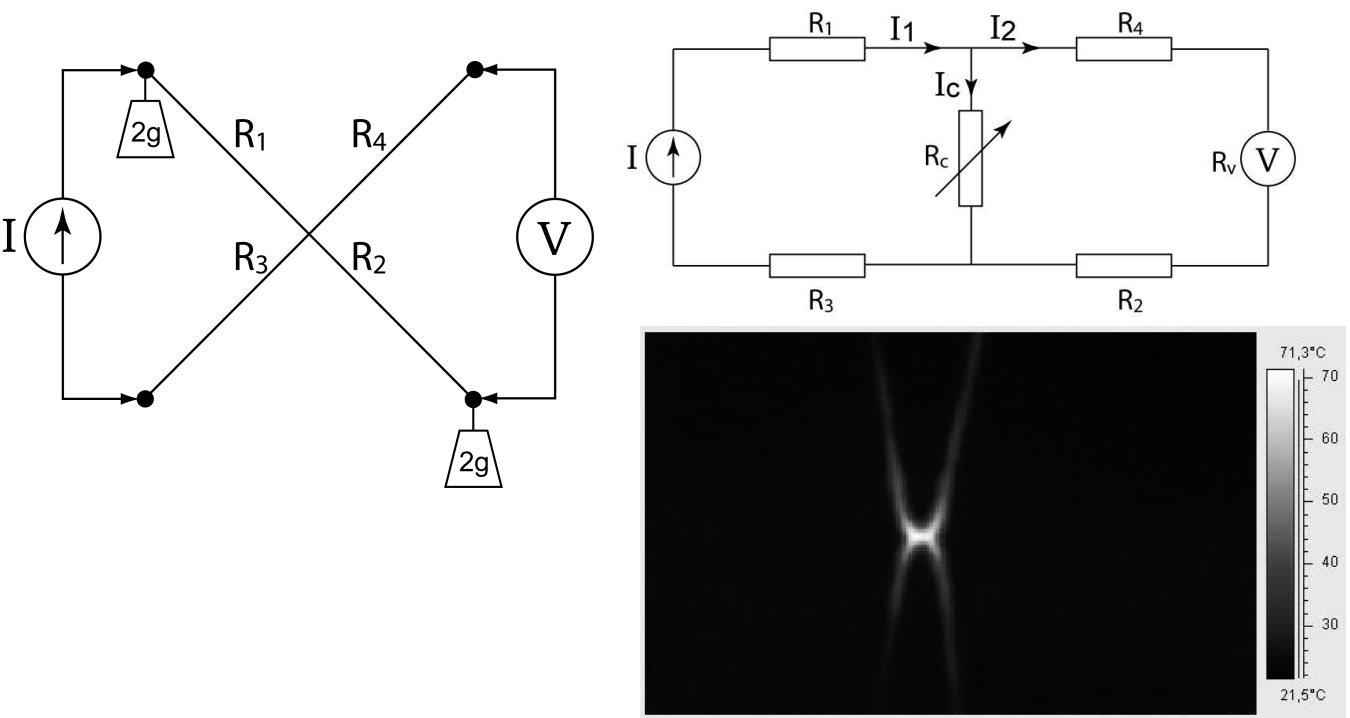

Fig. 9. : Contact resistance measurement setup (left), its equivalent electrical schematics (top right) and the thermogram of the contact point during the measurement (bottom right)

Two separate measurements were taken, between which the contact point was changed. During each measurement the current flowing through the yarns was increased from 0mA to $100 \mathrm{~mA}$ and then decreased from $100 \mathrm{~mA}$ to $0 \mathrm{~mA}$. The results of the measurements are depicted in the two graphs in Figs 10-11. In Fig. 10 for both measurements a very pronounced hysteresis is visible. To understand where the hysteresis comes from we need to remember that stainless steel is always covered with a thin passivation layer of chromium oxide $\left(\mathrm{Cr}_{2} \mathrm{O}_{3}\right)$ that prevents corrosion. For a sufficiently high current passing through the contact point the oxide layer between the yarns begins to burn out and the resistance suddenly drops, hence a hysteresis appears.

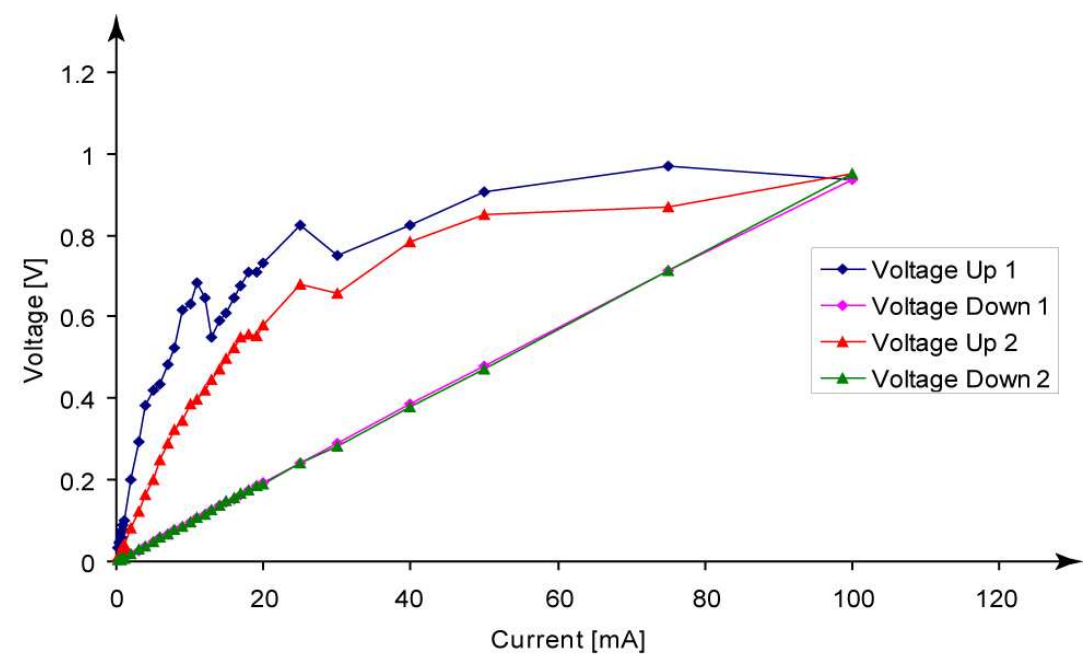

Fig. 10. : V-I characteristics for the contact point between two stainless steel yarns $(0-100 m A)$

Still, $100 \mathrm{~mA}$ flowing through a single yarn is a very high value, a magnitude that will never be reached in e.g. medical applications, where the total current flowing through the whole fabric is of the order of tens of $m A$. If so, then we are more interested in the behavior of the contact resistance for current values of a few mA. In Fig. 11 we show the same graph as before, but plotted only for currents up to $10 \mathrm{~mA}$. This part of the plot can be accurately approximated with a linear function, where the slope corresponds to an ohmic resistance. The reason 
is that for such small currents the power dissipated in the contact is too low for the chromium oxide to start burning out. Depending on the contact point and the respective thickness of the $\mathrm{Cr}_{2} \mathrm{O}_{3}$ layer we obtain a value of the contact resistance equal to $40 \Omega$ and $100 \Omega$, a result that fits accurately our previous estimations. Once the oxide is completely burned out the contact resistance becomes linear and equal to $10 \Omega$ for the whole measured range.

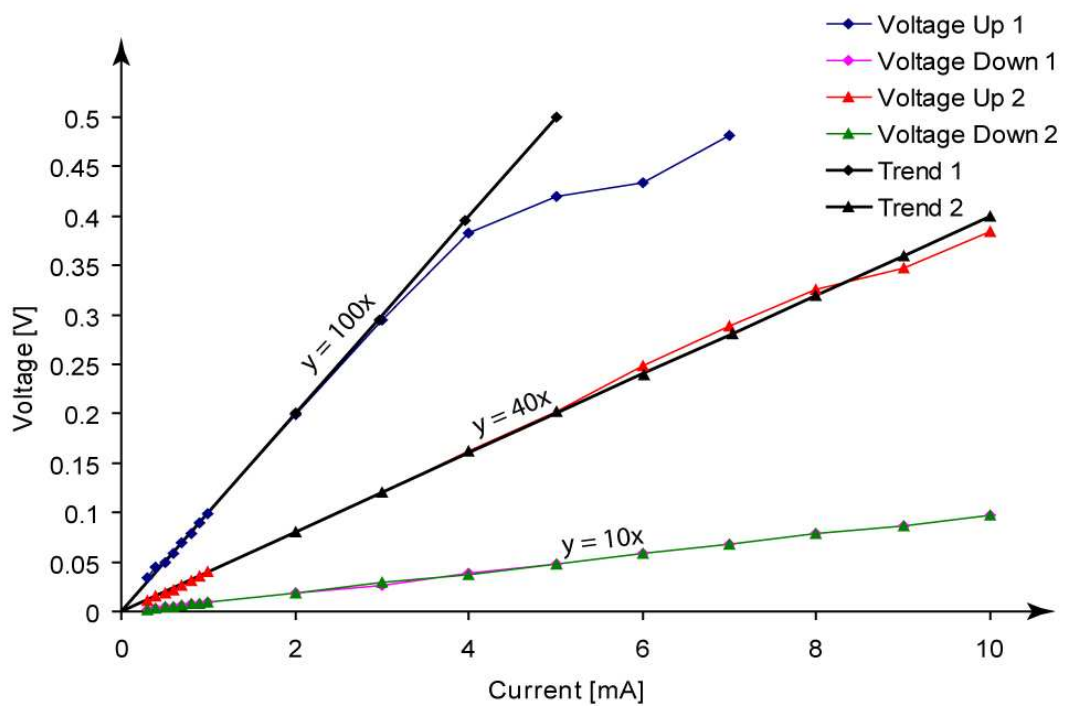

Fig. 11. : V-I characteristics for the contact point between two stainless steel yarns (0-10mA)

\section{Conclusions}

In our article we have presented an indirect and a direct method of measuring the contact resistance in electroconductive woven textiles. The first method is based on a comparison between infrared thermography, and a computer model of the power distribution in electroconductive fabrics, while the other one is a 4-point measurement. Both methods give very similar results and can be used complementarily. These results are also a very good verification of our model of current distribution in electroconductive textiles, which has been already presented in one of our earlier papers [5]. Furthermore, we found the contact resistance in Bekintex to be nonlinear, what we attribute to the burning out of the passivation layer of $\mathrm{Cr}_{2} \mathrm{O}_{3}$, which is always present on stainless steel. We are planning to continue our research on infrared thermography of electroconductive textiles with an emphasis on the design of electroconductive textile electrodes for blood flow electrostimulation.

\section{Acknowledgements}

This work has been done in the framework of the EU LIDWINE 026741-2 project. The financial support of the EU is gratefully acknowledged.

\section{REFERENCES}

[1] M. Lawrence, T. Kirstein, T. Keller, "Textile Electrodes for Transcutaneous Electrical Stimulation", EMPA conference talk, Dubendorf (Switzerland), 2004.

[2] I. Locher, M. Klemm, T. Kirstein, G. Troster, "Design and Characterization of Purely Textile Patch Antennas", Transactions on Advanced Packaging, vol. 29, No. 4, November 2006.

[3] I. Locher, G. Troster, "Fundamental Building Blocks for circuits on Textiles", IEEE Transactions on advanced packaging, vol. 30, NO. 3, AUGUST 2007.

[4] E. Bonderover, S. Wagner, "A Woven Inverter Circuit for e-Textile Applications", IEEE Electron Device Letters, vol. 25, No. 5, MAY 2004.

[5] J. Banaszczyk, G. De Mey, A Schwarz, L. Van Langenhove, "Current Distribution Modelling in Electroconductive Textiles", Proceedings of the $14^{\text {th }}$ International Conference MIXDES 2007, Ciechocinek, Poland, 21-23 June 2007, pp. 418 - 423

[6] S. P. Hersh, D. J. Mongomery, "Electrical Resistance Measurements on Fibers and Fiber Assemblies", Textile Research Journal, Vol. 22, No. 12, 805-818 (1952).

[7] J. Zieba, M. Frydrysiak, "Textronics-Electrical and Electronic Textiles. Sensors for Breathing Frequency Measurement", Fibres \& Textiles in Eastern Europe January/December 2006, Vol. 14, No. 5(59).

[8] K. W. Oh, H. J. Park, S. H. Kim, "Strechable Conductive Fabric for Electrotherapy", Journal of Applied Polymer Science, Vol. 88, 1225-1229 (2003). 
[9] K. A. Asanovic, T. A. Mihajlidi, S. V. Milosavljevic, D. D. Cerovic, J. R. Dojcilovic, "Investigation of the electrical properties of some textile materials", Journal of Electrostatics, Vol. 65, Issue 3, 162-167, March 2007.

[10] G. W. Collins, II, "Fundamental Numerical Methods and Data Analysis", pp. 44-46, http://ads.harvard.edu/books/1990fnmd.book/, 1990. 